\title{
EDITORIAL
}

\section{Natriuretic peptide receptors and the heart}

\section{King, M R Wilkins}

The synthesis of ANP and BNP by the heart has emerged as a sensor of cardiac strain, with increased synthesis the hallmark of myocyte hypertrophy in cell culture, and raised plasma concentrations a biomarker of ventricle dysfunction in vivo.

See end of article for authors' affiliations ...................

Correspondence to: Professor Martin R Wilkins, Section on Clinical Pharmacology, Faculty of Medicine, Imperial College, Hammersmith Hospital, Du Cane Road London W12 ONN, UK; m.wilkins@ic.ac.uk natriuretic peptide family. and other animals.
I is 20 years since de Bold reported that homogenates of normal atria stimulated a natriuresis and diuresis when administered intravenously to rats. ${ }^{1}$ This observation led to the isolation of atrial natriuretic peptide (ANP) from cardiac tissue. Subsequently other peptides with a similar ring structure and biological properties have been recognised, named in alphabetical order-BNP, CNP, and DNP-and termed the

Initial studies of the biology of natriuretic peptides focused on their endocrine role-measuring circulating levels in response to different stimuli and looking at the effects of administering exogenous peptide to isolated tissues and whole animals. Most attention has been given to ANP and BNP. Both are synthesised by cardiac myocytes and their production is increased by factors that increase cardiac work-pressure and volume overload. Indeed, the cardiac synthesis of ANP and BNP has emerged as a sensor of cardiac strain, with increased synthesis the hallmark of myocyte hypertrophy in cell culture and raised plasma concentrations a biomarker of ventricle dysfunction in vivo. Both peptides relax isolated resistance vessels. Pharmacological infusions of either peptide reduce renin and aldosterone concentrations, promote renal sodium and water excretion, and lower blood pressure in humans

These actions are mediated through specific receptors. Three main natriuretic peptide receptors (NPR) have been cloned. NPR-A (or GC-A) and NPR-B (or GC-B) are guanylyl cyclase linked and utilise cyclic GMP as the intracellular

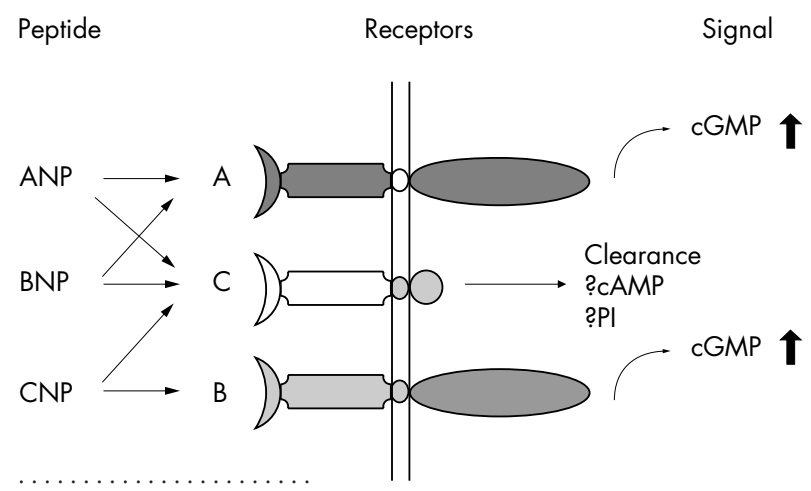

messenger. A third receptor, known as NPR-C, is not linked to guanylyl cyclase and may act to clear the natriuretic peptides from the circulation (fig 1). The rank order of potency of the peptides for NPR-A is ANP $\geqslant$ BNP $>>$ CNP, and for NPR-B it is $\mathrm{CNP}>\mathrm{ANP} \geqslant \mathrm{BNP}$. Consistent with a clearance role, NPR-C has less stringent structural requirements for binding, even tolerating D-amino acid substitutions.

The demonstration of NPRs in the heart encouraged speculation that these peptides may have a paracrine or indeed an autocrine role in the myocardium. Autoradiographic studies show that the main NPR subtype in the heart is NPR-C and is located in the endocardium. ${ }^{2}$ However, analysis of mRNA transcripts, western blotting, and functional studies indicate that NPRs are also found in cardiac fibroblasts and cardiac myocytes. ANP inhibits collagen synthesis in cardiac fibroblasts ${ }^{3}$ and both attenuates the growth response to adrenergic stimuli in cultured neonatal cardiac myocytes ${ }^{4}$ and induces apoptosis. ${ }^{5}$ This raises the possibility that, in addition to protecting the heart by reducing workload, ANP and BNP act directly on the myocardium to inhibit myocyte hypertrophy and fibrosis.

\section{PATHOPHYSIOLOGICAL ROLE OF NATRIURETIC PEPTIDES}

The classical approach to defining the pathophysiological role of a hormone in vivo is to inhibit its action. In the absence of discriminating receptor antagonists, the main approach in natriuretic peptide biology has been to "knock out" genes encoding ANP, BNP, and their receptors in mice. Homozygous NPR-A null mice are unequivocally hypertensive and, interestingly, the level of hypertension appears unaffected by reducing their salt intake. ${ }^{67}$ Further experiments in these mice have confirmed that NPR-A is the sole mediator of the vasorelaxant actions of ANP and BNP in systemic and pulmonary vessels and natriuresis-diuresis in the kidney. ${ }^{8-11}$ It would appear from these studies that NPR-A is an important mediator of the natriuresis of acute intravascular volume changes, but is dispensable for the regulation of sodium and water excretion in response to changes in dietary sodium intake. However, in another series of experiments, varying the expression of $\mathrm{Nprl}$, the gene encoding NPR-A, from one to four copies influenced blood pressure in a dose dependent manner. ${ }^{12}$ Mice with

Abbreviations: $A C E$, angiotensin converting enzyme; $A N P$, atrial natriuretic peptide; $B N P, B$ type natriuretic peptide; NEP, neutral endopeptidase; NPR, natriuretic peptide receptors 
below normal Nprl expression had salt sensitive hypertension while above normal expression exhibited lower blood pressure and were protected against high dietary salt.

Part of the phenotype of NPR-A "knockout" mice is cardiac hypertrophy.7 An early clue that this might be disproportionate to the degree of hypertension came in a report that male mice died prematurely (within six months) of cardiac causes. ${ }^{7}$ This extreme phenotype has been lost in ensuing generations, perhaps because of genetic drift and the loss of modifying loci. ${ }^{13}$ Subsequent more detailed studies have confirmed, however, that cardiac hypertrophy develops at an early age in NPR-A deficient mice and continues in the presence of effective antihypertensive treatment. Aortic banding to provide a fixed pressure-load on the heart produces a more pronounced degree of hypertrophy in mice lacking NPR-A than wild-type controls. ${ }^{13}$ The report by Kuhn and colleagues in this issue of Heart extends these findings p 000. ${ }^{14}$ These authors studied older animals, up to 12 months old, and observed that the chronic hypertension and cardiac hypertrophy is associated with more pronounced fibrosis and impaired cardiac contractility with advancing age, compared to wild-type controls. Taken together with the cell culture studies, the data indicate that NPR-A mediates a direct antihypertrophic action in the heart, independent of its role in regulating blood pressure. ${ }^{14}$

Given that NPR-A transduces both ANP and BNP activity, mice lacking this receptor cannot discriminate between the contributions of the two peptides, both of which are recruited by cardiac overload. Some insight here has come from ANP and BNP deficient mice. Homozygous ANP null mice are hypertensive and exhibit cardiac hypertrophy. ${ }^{15}$ Mice without BNP do not have hypertension or cardiac hypertrophy but are susceptible to cardiac fibrosis. ${ }^{16}$ There is also evidence that scar tissue formation is impaired in these mice following myocardial infarction, leading to rupture of the ventricle wall.

How can we make clinical use of this information? Enhancing the activity of natriuretic peptides has always been an attractive option for the treatment of hypertension and heart failure. The most practical route has been through inhibition of their metabolism by neutral endopeptidase (NEP), but clinical trialists were not impressed by the hypotensive or diuretic efficacy of NEP inhibitors when given alone. More recently molecules with dual NEP and angiotensin converting enzyme (ACE) inhibitory properties have been developed and are in clinical trials. One such drug, omapatrilat, is a potent antihypertensive agent and early reports suggest that it is effective in heart failure. We are already aware that ACE inhibitors attenuate cardiac hypertrophy and remodelling in hypertension and post-myocardial infarction. Data from studies in mice suggest that ANP and BNP are endogenous protec- tors of the myocardium. If this activity can be harnessed by NEP inhibitors, coupling NEP and ACE inhibition may be a powerful strategy for preserving cardiac structural integrity and function.

\section{ACKNOWLEDGEMENTS}

We are grateful for the support of the British Heart Foundation.

Authors' affiliations

L King, M R Wilkins, Section on Clinical Pharmacology, Faculty of Medicine, Imperial College, Hammersmith Hospital, London, UK

\section{REFERENCES}

1 de Bold AJ, Borenstein HB, Veress AT, et al. A rapid and potent natriuretic response to intravenous injection of atrial myocardial extract. Life Sci 1981;28:89-94.

2 Brown LA, Rutherford RA, Nunez DJ, et al. Downregulation of natriuretic peptide C-receptor protein in the hypertrophied ventricle of the aortovenocaval fistula rat. Cardiovasc Res 1997:36:363-71.

3 Redondo J, Bishop JE, Wilkins MR. Effect of atrial natriuretic peptide and cyclic GMP phosphodiesterase inhibition on collagen synthesis by adult cardiac fibroblasts. Br J Pharmacol 1998; 124:1455-62.

4 Horio T, Nishikimi T, Yoshihara F, et al. Inhibitory regulation of hypertrophy by endogenous atrial natriuretic peptide in cultured cardiac myocytes. Hypertension 2000;35:19-24.

5 Wu CF, Bishopric NH, Pratt RE. Atrial natriuretic peptide induces apoptosis in neonatal rat cardiac myocytes. J Biol Chem 1997;272:14860-6.

6 Lopez MJ, Wong SKF, Kishimoto I, et al. Salt-resistant hypertension in mice lacking the guanylyl cyclase-A receptor for atrial natriuretic peptide. Nature 1995;378:65-8

7 Oliver PM, Fox JE, Kim R, et al. Hypertension, cardiac hypertrophy, and sudden death in mice lacking natriuretic peptide receptor A. Proc Natl Acad Sci USA 1997:94:14730-5.

8 Dubois SK, Kishimoto I, Lillis TO, et al. A genetic model defines the importance of the atrial natriuretic peptide receptor (guanylyl cyclase-A) in the regulation of kidney function. Proc Natl Acad Sci USA 2000;97:4369-73.

9 Kishimoto I, Garbers DL, Dubois SK. The heart communicates with the kidney exclusively through the guanylyl cyclase-A receptor: acute handling of sodium and water in response to volume expansion. Proc Natl Acad Sci USA 1996;93:6215-19.

10 Lopez MJ, Garbers DL, Kuhn M. The guanylyl cyclase-deficient mouse defines differential pathways of natriuretic peptide signaling. J Biol Chem 1998;272:23064-8.

11 Zhao L, Long L, Morrell NW, et al. NPR-A-Deficient mice show increased susceptibility to hypoxia-induced pulmonary hypertension. Circulation 1999:99:605-7.

12 Oliver PM, John SWM, Purdy KT, et al. Natriuretic peptide receptor expression influences blood pressures of mice in a dose-dependent manner. Proc Natl Acad Sci USA 1998;95:2547-51.

13 Knowles JW, Esposito G, Mao L, et al. Pressure-independent enhancement of cardiac hypertrophy in natriuretic peptide receptor A-deficient mice. J Clin Invest 2001;107:975-84.

14 Kuhn N, Holtwick R, Baba HA, et al. Progressive cardiac hypertrophy and dysfunction in atrial natriuretic peptide receptor (GC-A) deficient mice. Heart 2002;87: IN THIS ISSUE.

15 John SWM, Krege JH, Oliver PM, et al. Genetic decreases in atrial natriuretic peptide and salt-sensitive hypertension. Science 1995;267:679-81

16 Tamura N, Ogawa Y, Chusho H, et al. Cardiac fibrosis in mice lacking brain natriuretic peptide. Proc Natl Acad Sci USA 2000;97:4239-44.

\section{STAMPS IN CARDIOLOGY}

\section{Myocardial infarction}

The campaign against heart disease was the theme of National Health Day for Italy in 1993. The stamp issued featured myocardial infarction and shows a wedge stopping a heart shaped cog-the design being repeated in the cancellation marks on the first day cover. The stamp is inscribed "lotta contro l'infarto".

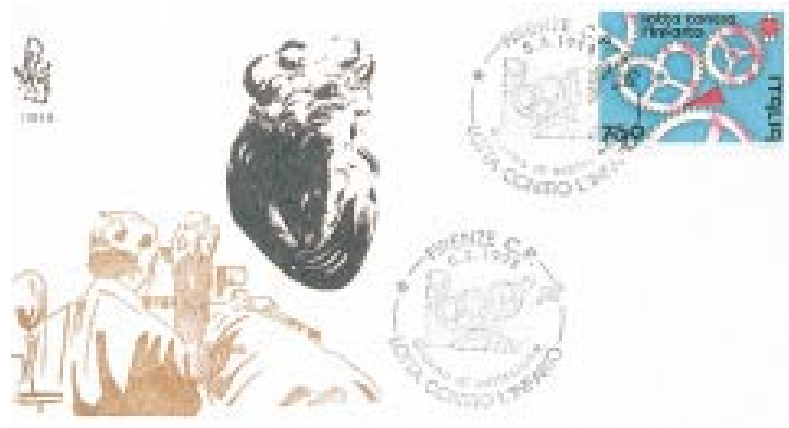

M K Davies A Hollman 